\title{
An Eminent Absence: Agenesis of Inferior Vena Cava Underlying Bilateral Iliac Vein Thrombosis
}

\author{
Giorgia Protti, Fabrizio Elia, Francesca Bosco, Franco Aprà \\ High Dependency Unit, San Giovanni Bosco Hospital, Torino, Italy
}

Received: 14/09/2020

Accepted: 28/09/2020

Published: $22 / 10 / 2020$

\begin{abstract}
How to cite this article: Protti G, Elia F, Bosco F, Aprà F. An eminent absence: agenesis of inferior vena cava underlying bilateral iliac vein thrombosis.
\end{abstract} EJCRIM 2020;7: doi:10.12890/2020_001999.

Conflicts of Interests: The Authors declare that there are no competing interests.

This article is licensed under a Commons Attribution Non-Commercial 4.0 License

\section{ABSTRACT}

Among thrombophilic risk factors for deep venous thrombosis (DVT), agenesis of the inferior vena cava (AIVC) is very rare, but it must be considered in specific settings. Here, we present the case of an 18-year-old woman who was admitted to the Emergency Department with swelling and pain of her left leg. Clinical examination and ultrasonography detected extensive proximal DVT of the left leg. After attempted mechanical thrombectomy failed, an abdominal CT scan was obtained, which demonstrated bilateral thrombosis of the iliac-femoral axis in the context of congenital AIVC.

\section{LEARNING POINTS}

- Agenesis of the inferior vena cava is a rare risk factor for deep venous thrombosis.

- Agenesis of the inferior vena cava requires lifetime follow-up and treatment.

- Idiopathic deep venous thrombosis, especially in young patients, requires additional diagnostic investigation that considers congenital anomalies.

\section{KEYWORDS}

Inferior vena cava, agenesis, deep venous thrombosis, thrombophilia

\section{INTRODUCTION}

Agenesis of the inferior vena cava (AIVC) is an uncommon congenital condition resulting from a defect during embryogenesis. Since it was detected, it has been reported to be associated with deep venous thrombosis (DVT), especially in young people. We report a case of a young woman presenting with DVT of both iliac veins, and the subsequent accidental finding of agenesis of the infrarenal segment of the inferior vena cava (IVC).

\section{CASE DESCRIPTION}

An 18-year-old woman presented to the Emergency Department after the sudden onset of swelling and pain of her left leg. Her medical history was substantially negative. She reported occasional smoking and taking oral contraceptives as the only home medication. Recent months were notable for the occurrence of lumbar pain (which the patient ascribed to dysmenorrhoea).

On examination, the whole left leg was found to be swollen, tense and painful to palpation; no tortuous or palpable veins were found; no sensory-motor deficits were observed. DVT was diagnosed clinically, and ultrasonography demonstrated extensive thrombosis of the external iliac, common femoral and the proximal segment of the superficial femoral vein on the left side, showing no DVT with regards to the right system and the IVC. A CT scan of the chest was performed, which ruled out thromboembolism of the lungs. 
Anticoagulant therapy with fondaparinux was initiated. An attempt at mechanical thrombectomy through right common femoral vein access was made, but the procedure had to be interrupted due to the finding of obstruction of the right iliac vein with multiple hypertrophic collateral lumbar and vertebral veins converging in the azygos vein, which was hypertrophic itself. At that point, a CT scan of the abdomen was performed, showing thrombosis of both the left and right iliac-femoral axes (Fig. 1), partial thrombosis of the IVC, which appeared to be small and consistent with a condition of agenesis (Fig. 2A); the lumbar veins bilaterally also appeared to be partially thrombotic; ectasia of the vertebral venous plexus and the azygos-hemiazygos system was seen (Fig. 2B).

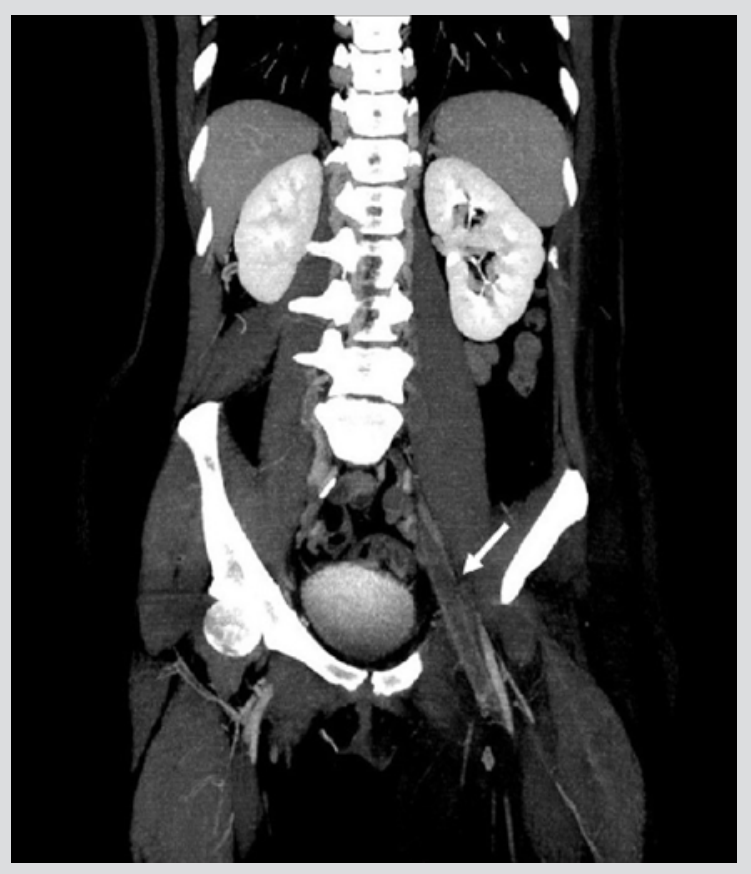

Figure 1. Total thrombotic obliteration of the left common iliac axis (white arrow)

Figure 2. IVC not visible in its usual position in A (white arrow); ectasia of azygos (thin arrow) and hemiazygos (thick arrow) system in B
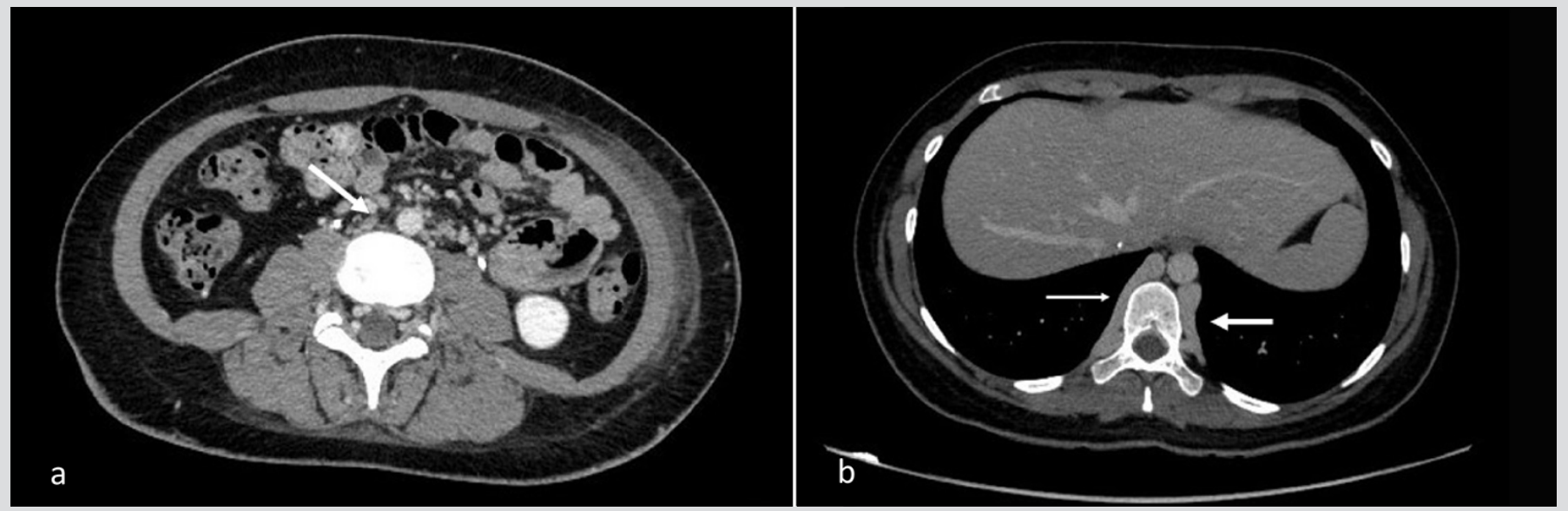

In consideration of the poor clinical improvement after 15 days of anticoagulant therapy, another attempt to favour recanalization was made, entering through the common femoral vein on the left side, and we managed to aspirate $250 \mathrm{ml}$ of thrombotic material, partially recanalizing the left femoral-iliac axis. After the procedure, oral anticoagulation with warfarin was started, and a haematological follow-up was planned after discharge.

\section{DISCUSSION}

Although DVT is a common clinical presentation, AIVC is believed to be a very rare risk factor, with an estimated prevalence of $0.0005-1 \%$ in the general population ${ }^{[1-3]}$. 
The embryogenesis of the IVC occurs between weeks 6 and 8 of gestation from 3 separate sets of paired veins that merge to form the IVC. At least 2 embryogenetic theories have been advanced for AIVC, the first proposing a primary congenital anomaly of IVC, the second suggesting a perinatal thrombosis causing regression of a previously normal IVC. The hypothesized cause of DVT in these patients is that, despite the development of collateral venous systems, venous drainage of the lower limbs is inadequate, leading to venous stasis and clotting ${ }^{[4]}$.

Some common features of patients with AIVC presenting with DVT are young age (mean age of 30 years) and male sex prevalence; the thrombosis preferentially involves the iliac and femoral veins, often bilaterally, and usually occurs after exposure to a precipitating factor (physical trauma or injury, prolonged immobility, use of birth control pills, thrombophilic coagulation disorders) ${ }^{[1,2]}$. Collateral ectatic veins are present, mostly draining into the azygos and hemiazygos veins. Pulmonary embolism is a rare complication of DVT in AIVC, probably due to the small diameter of the vessels leading to the pulmonary circulation in the absence of the IVC ${ }^{[2]}$. In the vast majority of cases, AIVC is thoroughly asymptomatic, and is detected as an incidental finding during imaging studies for other purposes. The most common symptoms, apart from those related to DVT, are due to the increased pressure and congestion of the upstream veins: venous insufficiency of the legs (with varicose veins and venous ulcers), haematuria, varicocele in males and pelvic congestion syndrome in females ${ }^{[2]}$.

Conventional ultrasonography has a limited role in detecting AIVC, as intra-abdominal vascular abnormalities are difficult to identify with this technique ${ }^{[1,2]}$. CT scans with intravenous contrast and MRI of the abdomen are highly sensitive, even if these are usually performed in order to rule out other diseases (for example, abdominal masses or malignancies) ${ }^{[2]}$. Venography is the gold standard for imaging diagnosis, but it is also highly invasive ${ }^{[4]}$.

Given the limited number of cases, no clear consensus has been reached on therapeutic strategy other than long-term anticoagulation and wearing elastic stockings. As AIVC is a permanent condition and a lifelong risk factor for recurrent DVT, prolonged oral anticoagulation should perhaps be prescribed indefinitely, planning frequent evaluation of the related haemorrhagic risk. Since no studies exist concerning the utilization of DOACs in this unusual context, warfarin has been recommended for anticoagulant therapy thus far ${ }^{[1,2,4]}$. Elastic stocking support and leg elevation are suggested, as well as avoidance of additional risk factors ${ }^{[2]}$. Some authors have reported performing thrombolysis/mechanical thrombectomy in addition to anticoagulation, but these results are inconsistent. A surgical approach has been reserved for nonhealing venous ulcers, by positioning a prosthetic bypass graft from the iliac to the intrathoracic azygos vein ${ }^{[1]}$. Surgical reconstruction of the IVC has been reported, and the long-term benefits remain to be seen.

\section{CONCLUSION}

AIVC is an uncommon risk factor for DVT. Nevertheless, it should be kept in mind in cases of young patients with idiopathic DVT of the proximal lower limbs. Given the rarity of this condition, no specific guidelines exist regarding treatment, but anticoagulation is probably needed for life. Where AIVC is detected, it is important to instruct the patient about his/her increased thrombophilic risk, how to avoid precipitating factors and to seek immediate medical help if evocative symptoms arise.

\section{REFERENCES}

1. O'Connor DB, O'Brien N, Khani T, Sheehan S. Superficial and deep vein thrombosis associated with congenital absence of the infrahepatic inferior vena cava in a young male patient. Ann Vasc Surg 2011;25(5):697.e1-e4.

2. Lambert M, Marboeuf P, Midulla M, Trillot N, Beregi JP, Mounier-Vehier C, et al. Inferior vena cava agenesis and deep vein thrombosis: 10 patients and review of the literature. Vasc Med 2010;15(6):451-459.

3. Ruggeri M, Tosetto A, Castaman G, Rodeghiero F. Congenital absence of the inferior vena cava: a rare risk factor for idiopathic deep-vein thrombosis. Lancet 2001;357(9254):441.

4. Obernosterer A, Aschauer M, SchnedI W, Lipp RW. Anomalies of the inferior vena cava in patients with iliac venous thrombosis. Ann Intern Med 2002;136(1):37. 